\title{
Clinical Reasoning: A 42-year-old man with unilateral leg weakness
}

Raphael Schneider, MD, Anna Weichert, PhD, Ekaterina Rogaeva, PhD, Janice Robertson, PhD, Julia Keith, PhD,* and Lorne Zinman, MD, MSc*

Neurology ${ }^{\circledR}$ 2018;90:e1085-e1090. doi:10.1212/WNL.0000000000005179

\section{Section 1}

A previously healthy 42 -year-old man, with a family history of muscle weakness in an older brother and a paternal uncle, developed proximal right leg weakness. He had first noticed slowness in running followed by difficulty climbing stairs and rising from low surfaces. $\mathrm{He}$ unintentionally lost about 10 pounds. When he was assessed 3 months after symptom onset, he had also developed proximal limb weakness and fasciculations in both arms. He denied dysarthria, dysphagia, and respiratory symptoms. He had no sensory complaints. On physical examination, cranial nerves were normal. There was diffuse loss of muscle bulk in the upper and lower limbs affecting proximal muscle groups and intrinsic hand muscles. Tone was normal. There was widespread proximal and distal muscle weakness. Deep tendon reflexes were absent for brachioradialis tendons, the right patella, and Achilles tendons, but otherwise normal. Plantar responses were downgoing. Coordination and sensory examination were normal.

\section{Questions for consideration:}

1. Where would you localize the disease process?

2. What additional testing would you recommend at this point?
Correspondence Dr. Schneider raphael.schneider@ utoronto.ca

GO TO SECTION 2

\footnotetext{
*These authors contributed equally to this work.
}

From the Department of Medicine, Division of Neurology (R.S., L.Z.), and Department of Anatomical Pathology (J.K.), Sunnybrook Health Sciences Centre, and Tanz Centre for Research in Neurodegenerative Disease (R.S., A.W., E.R., J.R.), University of Toronto, Canada.

Go to Neurology.org/N for full disclosures. Funding information and disclosures deemed relevant by the authors, if any, are provided at the end of the article. 


\section{Section 2}

This patient has a fairly rapidly progressing motor syndrome. At this point, the differential diagnosis includes conditions that affect the motor neuron, the nerve, and the muscle. In the context of his family history, one should think of familial forms of motor neuron disease, such as familial amyotrophic lateral sclerosis (fALS). The predominantly lower motor neuron pattern would also fit with progressive muscular atrophy (PMA). Despite the absence of bulbar symptoms, perioral or tongue fasciculations, and gynecomastia, the Xlinked recessive Kennedy disease (or spinobulbar muscular atrophy) should be considered in this male patient with a family history of muscle weakness in adult male relatives. ${ }^{1}$ Multifocal motor neuropathy (MMN) is another disease that presents as a pure motor syndrome. However, MMN classically affects distal muscles and progresses slowly. Occasionally, motor-predominant chronic inflammatory demyelinating polyneuropathy (CIDP) can present with weakness only. However, fluctuation of symptoms and mild sensory findings are typically seen even in motor-predominant CIDP. Hereditary myopathies, such as myotonic dystrophy, can also present with slowly progressive proximal and distal limb weakness. Typically, myotonic dystrophy presents with a combination of weakness and atrophy of facial and jaw muscles, drooping eyelids, and frontal hair loss. Acquired myopathies, and specifically inclusion body myositis (IBM), should be considered in patients with proximal lower limb weakness. However, the patient's disease onset before 50 years of age makes IBM a relatively unlikely diagnosis.

Bloodwork and electrophysiologic studies were ordered. The patient's bloodwork was normal, including normal creatine kinase (CK). Nerve conduction studies (NCS) showed decreased motor amplitudes without evidence of conduction block (CB) or dispersion. Conduction velocities were normal. Sensory nerve action potentials (SNAPs) were normal. EMG showed fibrillations, fasciculation, large motor units, and decreased recruitment consistent with chronic and active denervation in the right cervical and lumbosacral regions. The patient was unable to tolerate needle examination of the paraspinal muscles.

\section{Questions for consideration:}

1. What is the significance of the test results?

2. What additional testing would you order at this point?

GO TO SECTION 3 


\section{Section 3}

The combination of decreased motor amplitudes on NCS and needle EMG showing fasciculations, large motor units, and decreased recruitment supports a process affecting the motor neurons. In Kennedy disease, SNAPs can be normal, but are often reduced. The absence of slowing and $\mathrm{CB}$ on NCS is an important piece of information. Since CB in motor nerve fibers is the hallmark of MMN, this diagnosis becomes less likely. It is important to note, however, that some patients with otherwise typical MMN have no CB and that $\mathrm{MMN}$ should not be mistaken for motor neuron disease since MMN is treated differently. ${ }^{2}$ To differentiate between these conditions, one needs to keep in mind that in MMN the muscle deficit corresponds to a nerve distribution and needle EMG shows no diffuse denervation. Normal conduction velocities and no evidence of CB make CIDP unlikely. The normal CK puts myopathy low on the list of potential diagnoses.

\section{Questions for consideration:}

1. How is a diagnosis of motor neuron disease made?

2. What additional testing would you order at this point?

GO TO SECTION 4 


\section{Section 4}

Motor neuron disease remains a diagnosis of exclusion. The El Escorial Criteria for the diagnosis of amyotrophic lateral sclerosis (ALS) ${ }^{3}$ define 4 body regions to be assessed by the clinician: brainstem, cervical, thoracic, and lumbosacral. According to these criteria, a definite diagnosis of ALS can be made when upper and lower motor neuron signs are detected in 3 regions. EMG can demonstrate the involvement of the lower motor neuron in affected and frequently also in clinically unaffected regions. The criteria also require imaging studies to rule out structural lesions of the brain and spinal cord, which can mimic some of the abovementioned findings. Especially cervical spondylotic myelopathies can mimic upper motor neuron pathology. In our patient, CT head and MRI spine did not show any significant abnormalities.

\section{Questions for consideration:}

1. Based on these findings, what is your current differential diagnosis?

2. What further testing would you perform to confirm the diagnosis?

GO TO SECTION 5 


\section{Section 5}

The history and examination is consistent with motor neuron disease with a predominantly lower motor neuron pattern. At this point, fALS with a predominantly lower motor neuron pattern, PMA, and Kennedy disease remain diagnostic considerations. There has been some debate about whether PMA is an early manifestation of ALS. A large study of patients with
PMA showed that a substantial proportion develops upper motor neuron signs over time and that PMA should be viewed as part of the ALS spectrum rather than as a distinct entity. ${ }^{4}$ Patients with Kennedy disease typically develop bulbar symptoms due to lower motor neuron involvement in the 4th or 5th decade of life. Kennedy disease is inherited in an $\mathrm{X}$-linked recessive pattern and hence genetic testing can help

Figure Histopathologic features of the presented case
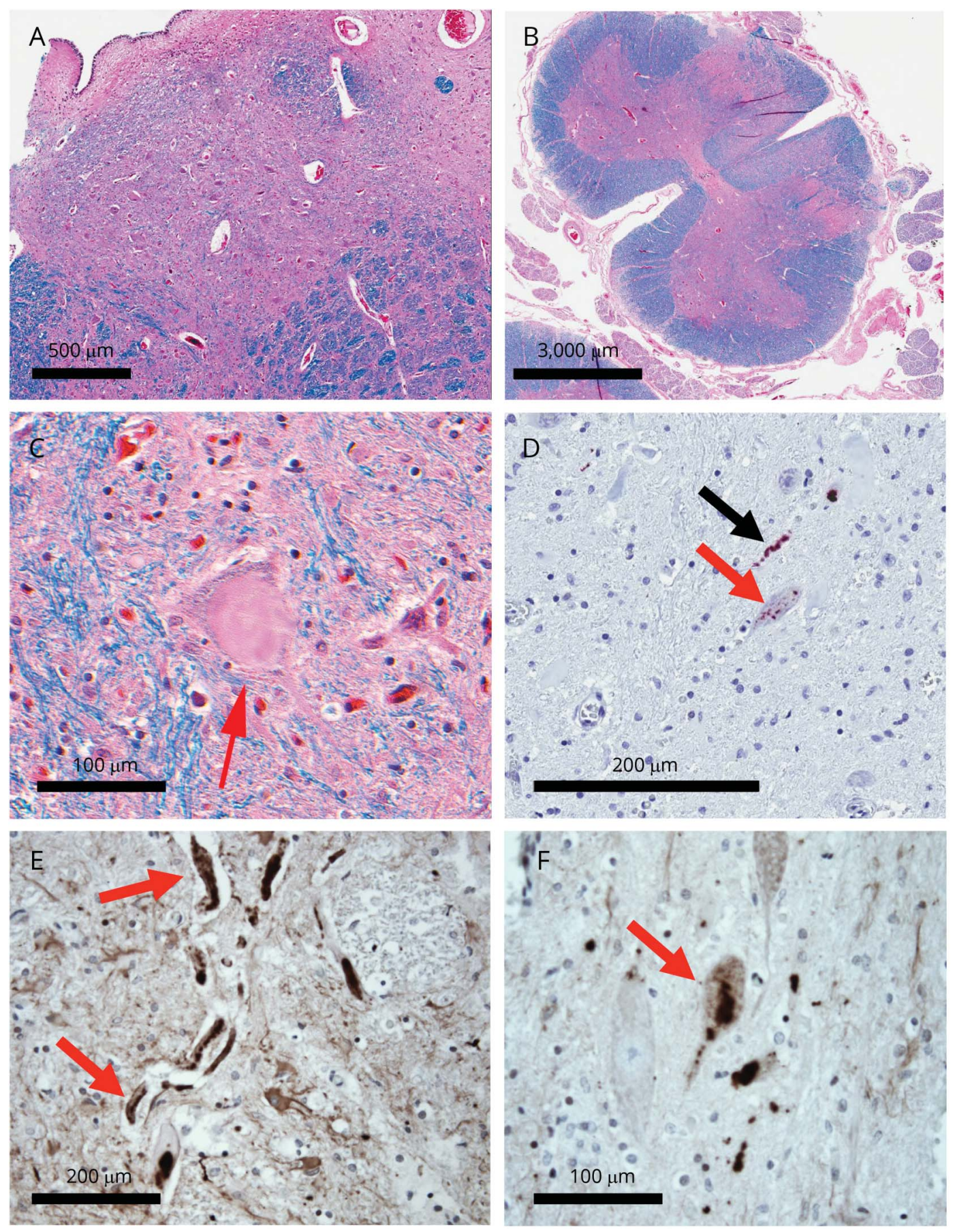

H\&E stain of the hypoglossal nucleus (A) and lumbar spinal cord (B) shows a reduced population of motor neurons. Hyaline conglomerate inclusion in a motor neuron is shown (C, red arrow). p62 immunopositive inclusions that are axonal ( $D$, black arrow) and in the neuronal cytoplasm ( $D$, red arrow) are shown. SOD1-immunopositive inclusions in axons labeled with antibody to misfolded SOD1 (SEDI antibody) (E, red arrows) and in a motor neuron cell body ( $F$, red arrow) are shown. 
support the diagnosis. ${ }^{1}$ However, in our patient, genetic testing conducted as described previously ${ }^{5}$ revealed a pathologic mutation in the copper zinc superoxide dismutase 1 (SOD1) p.A4V (NM_000454:exon1:c.C14T:p.A5V), consistent with a diagnosis of fALS. The patient died of respiratory failure 7 months after his first symptoms had been noticed. At autopsy, there was marked selective neuronal loss from the motor cranial nerve nuclei and anterior horns (figure), as well as degeneration of the corticospinal and spinocerebellar tracts. Some remaining motor neurons contained cytoplasmic inclusions; some were hyaline conglomerate inclusion (figure). The motor cortex, dorsal columns, and cerebellum were essentially normal, and there was no associated frontotemporal lobar degeneration. Additional immunohistochemistry showed that the inclusions were p62positive (figure) and positive for misfolded SOD1 (figure) but negative for all other markers employed, including transactive response DNA binding protein 43 (TDP-43) and fused in sarcoma (FUS) (data not shown).

\section{Discussion}

Strictly speaking, the patient's clinical presentation alone did not fulfill the El Escorial Criteria for the diagnosis of ALS. However, genetic testing and postmortem pathologic analysis was consistent with a diagnosis of ALS, underlining the value of these investigations in making an accurate diagnosis. Approximately $10 \%$ of patients with ALS have one of the familial forms of the disease. Mutations in various genes including SOD1, TARDBP (encoding for TDP-43), FUS, and chromosome 9 open reading frame 72 (C9orf72), can cause fALS. ${ }^{6}$ Notably, the patient was negative for the C9orf72 repeat expansion (genotype 2/8), and genome-wide array (NeuroX; Illumina, San Diego, CA) designed to investigate neurodegenerative diseases did not detect any additional known pathogenic mutations in 77 genes implicated in these disorders. ${ }^{7}$ Within the fALS subgroup, about $20 \%$ of patients carry a mutation in SOD1 and exhibit hyaline conglomerate inclusions containing misfolded SOD $1 .^{8}$ The patient's SOD1 mutation, alanine to valine substitution in codon 4 of exon 1 (p.A4V; GCC>GTC), is responsible for about 50\% of SOD1 mutations associated with ALS in North America. It appears the mutation has had 2 distinct origins in the course of human evolution, one in Europe and the other in Amerindians. ${ }^{9}$ The inheritance pattern is autosomal dominant and many patients have a positive family history of motor neuron disease. Like our patient, affected individuals usually present at a relatively young age with predominant lower motor neuron involvement. Many patients with ALS have some cognitive impairment (20\%-50\%), and ALS co-occurs with frontotemporal dementia in about $15 \%$ of patients with ALS. ${ }^{6}$ Data on cognitive symptoms in SOD1 fALS is sparse; one study showed that patients with SOD1 fALS rarely have cognitive symptoms. ${ }^{10}$ Mild corticospinal tract involvement and severe degeneration of the lower motor neurons are features of SOD1 fALS pathology ${ }^{\mathrm{e}}$ (links.lww.com/WNL/A263). We observed abundant and thick neuronal inclusions that were SOD1-immunopositive.

ALS is difficult to treat and there is no cure. Therapeutic approaches aimed at correcting mutations or their detrimental effects hold great promise for patients with fALS. Antisense oligonucleotide therapy has shown to be successful in animal models of SOD1 $\mathrm{ALS}^{\mathrm{e} 2}$ and several treatment trials in humans are currently underway (clinicaltrials.gov). Identifying disease-causing mutations in patients with ALS will therefore become even more critical and may influence treatment decisions in the near future.

\section{Author contributions}

R.S., A.W., E.R., J.R., J.K., and L.Z. made substantial contributions to the study design and analysis of data. RS drafted the manuscript. A.W., E.R., J.R., J.K., and L.Z. revised the manuscript critically for important intellectual content and gave final approval of the version to be published.

\section{Study funding}

No targeted funding reported.

\section{Disclosure}

R. Schneider holds an ALS Canada Clinical Research Fellowship. A. Weichert, E. Rogaeva, J. Robertson, J. Keith, and L. Zinman report no disclosures relevant to the manuscript. Go to Neurology.org/N for full disclosures.

\section{References}

1. Kennedy WR, Alter M, Sung JH. Progressive proximal spinal and bulbar muscular atrophy of late onset: a sex-linked recessive trait. Neurology 1998;50:583-593.

2. Delmont E, Azulay JP, Giorgi R, et al. Multifocal motor neuropathy with and without conduction block: a single entity? Neurology 2006;67:592-596.

3. Brooks BR, Miller RG, Swash M, Munsat TL. El Escorial revisited: revised criteria for the diagnosis of amyotrophic lateral sclerosis. Amyotroph Lateral Scler Other Motor Neuron Disord 2000;1:293-299.

4. Kim WK, Liu X, Sandner J, et al. Study of 962 patients indicates progressive muscular atrophy is a form of ALS. Neurology 2009; 73:1686-1692.

5. Zinman L, Liu HN, Sato C, et al. A mechanism for low penetrance in an ALS family with a novel SOD1 deletion. Neurology 2009;72:1153-1159.

6. Ling SC, Polymenidou M, Cleveland DW. Converging mechanisms in ALS and FTD disrupted RNA and protein homeostasis. Neuron 2013;79:416-438.

7. Ghani M, Lang AE, Zinman L, et al. Mutation analysis of patients with neurodegenerative disorders using NeuroX array. Neurobiol Aging 2015;36:545 e549-514.

8. Liu HN, Sanelli T, Horne P, et al. Lack of evidence of monomer/misfolded superoxide dismutase-1 in sporadic amyotrophic lateral sclerosis. Ann Neurol 2009;66: $75-80$.

9. Saeed M, Yang Y, Deng HX, et al. Age and founder effect of SOD1 A4V mutation causing ALS. Neurology 2009;72:1634-1639.

10. Wicks P, Abrahams S, Papps B, et al. SOD1 and cognitive dysfunction in familial amyotrophic lateral sclerosis. J Neurol 2009;256:234-241. 


\section{Neurology}

\section{Clinical Reasoning: A 42-year-old man with unilateral leg weakness}

Raphael Schneider, Anna Weichert, Ekaterina Rogaeva, et al.

Neurology 2018;90;e1085-e1090

DOI 10.1212/WNL.0000000000005179

This information is current as of March 19, 2018

\section{Updated Information \&} Services

References

Subspecialty Collections

Permissions \& Licensing

Reprints including high resolution figures, can be found at: http://n.neurology.org/content/90/12/e1085.full

This article cites 10 articles, 5 of which you can access for free at: http://n.neurology.org/content/90/12/e1085.full\#ref-list-1

This article, along with others on similar topics, appears in the following collection(s):

\section{All Genetics}

http://n.neurology.org/cgi/collection/all_genetics Amyotrophic lateral sclerosis

http://n.neurology.org/cgi/collection/amyotrophic_lateral_sclerosis_

Information about reproducing this article in parts (figures,tables) or in its entirety can be found online at:

http://www.neurology.org/about/about_the_journal\#permissions

Information about ordering reprints can be found online:

http://n.neurology.org/subscribers/advertise

Neurology ${ }^{\circledR}$ is the official journal of the American Academy of Neurology. Published continuously since 1951, it is now a weekly with 48 issues per year. Copyright (O 2018 American Academy of Neurology. All rights reserved. Print ISSN: 0028-3878. Online ISSN: 1526-632X.

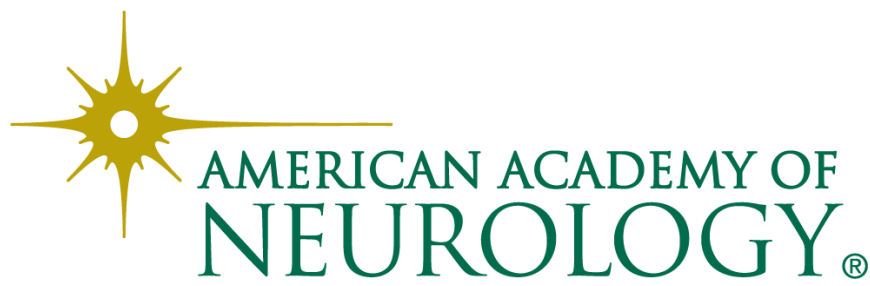

